\title{
IMWG Flow MRD-negative
}

National Cancer Institute

\section{Source}

National Cancer Institute. IMWG Flow MRD-negative. NCI Thesaurus. Code C159801.

Absence of phenotypically aberrant clonal plasma cells by next-generation flow cytometry on bone marrow aspirates using the EuroFlow standard operation procedure for minimal residual disease detection in multiple myeloma (or validated equivalent method) with a minimum sensitivity of 1 in $10^{\wedge} 5$ nucleated cells or higher. 